\title{
BMJ Open Factors associated with return to work among people on work absence due to long-term neck or back pain: a narrative systematic review
}

Mamunur Rashid, ${ }^{1}$ Marja-Leena Kristofferzon, ${ }^{2,3}$ Annika Nilsson, ${ }^{2,3}$ Marina Heiden ${ }^{1}$

To cite: Rashid M, Kristofferzon M-L, Nilsson A, et al. Factors associated with return to work among people on work absence due to long-term neck or back pain: a narrative systematic review. BMJ Open 2017;7:e014939. doi:10.1136/ bmjopen-2016-014939

- Prepublication history and additional material are available. To view these files please visit the journal online(http://dx.doi. org/10.1136/bmjopen-2016014939)

Received 27 0ctober 2016 Revised 11 April 2017 Accepted 24 April 2017

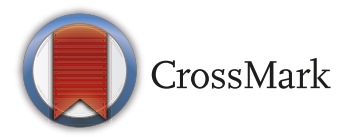

${ }^{1}$ Department of Occupational and Public Health Sciences, Centre for Musculoskeletal Research, Faculty of Health and Occupational Studies, University of Gävle, Gävle, Sweden

${ }^{2}$ Department of Health and Caring Sciences, Faculty of Health and Occupational Studies, University of Gävle, Gävle, Sweden

${ }^{3}$ Department of Public Health and Caring Sciences, Faculty of Medicine, Uppsala University, Uppsala, Sweden

Correspondence to

Mr Mamunur Rashid; mamunur. rashid@hig.se

\section{ABSTRACT}

Objective The purpose of this narrative systematic review was to summarise prognostic factors for return to work (RTW) among people with long-term neck/shoulder or back pain.

Methods A systematic literature search was performed through three databases (Medline, CINAHL and PsycINF0) for studies published until February 2016. Only observational studies of people on work absence (â\%॰ $¥ 2$ weeks) due to neck/shoulder or back pain were included. The methodological quality of the included studies was assessed using guidelines for assessing quality in prognostic studies on the basis of Framework of Potential Biases. Factors found in the included studies were grouped into categories based on similarities and then labelled according to the aspects covered by the factors in the category.

Results Nine longitudinal prospective cohort studies and one retrospective study fulfilled the inclusion criteria. From these, five categories of factors were extracted. Our findings indicate that recovery beliefs, health-related factors and work capacity are important for RTW among people with long-term neck or back pain. We did not find support for workplace factors and behaviour being predictive of RTW.

Conclusions Our findings suggest that recovery beliefs, perceived health and work capacity may be important targets of intervention for people with long-term neck or back pain. However, more high-quality prospective studies are needed to confirm the results and improve our understanding of what is needed to facilitate RTW in this population.

\section{INTRODUCTION}

Work absence due to musculoskeletal disorders (MSDs) is a considerable public health problem, as it results in workers' compensation, medical expense as well as productivity loss. ${ }^{1-5}$ Low back pain (LBP) is considered one of the leading causes of work absence $e^{6-9}$ and neck/shoulder disorders are also among most common reasons for being absent from work. ${ }^{10-12}$ Despite improvements in objective measures of health, work absence has increased substantially in western European

\section{Strengths and limitations of this study}

- The review identifies prognostic factors of importance for the natural course of return to work in people with long-term pain.

- Findings are based on quality-assessed studies considering areas of potential bias.

- Few high-quality studies prevent solid conclusions about prognostic factors in this population.

- Since most studies concerned low back pain only, the results are less applicable to people with neck pain.

countries. ${ }^{4}{ }^{13-15}$ It may be that workers have difficulties returning to work for reasons other than their health condition, for instance psychosocial problems. ${ }^{16-19}$

It follows that return to work (RTW) following MSDs is a complex process that is not solely dependent on physical ability. Studies have shown that it may also be affected by recovery beliefs, pain-related behaviours, work-related factors and health-related factors, ${ }^{17}{ }^{20-29}$ but the results differ considerably between studies. Reasons for this may be heterogeneous samples, differences in measurements and the analytical strategy used for identifying the factors. Moreover, many studies have been conducted to identify risk factors for work absence, ${ }^{16}{ }^{30-33}$ but their results cannot be readily inverted to indicate what is needed for people to RTW. To reduce work absence among people with MSDs, it is important to know which factors are important for RTW, and thereby what should be prioritised in the treatment/rehabilitation.

To date, most empirical studies on pain and RTW have focused on acute pain and LBP. $^{31}{ }^{33-35}$ Accordingly, most of previous systematic reviews addressing factors of importance for RTW have focused on people with acute pain. For example, Steenstra $e t a t^{20}$ 
found that workers' recovery expectations are important factors affecting the likelihood and timing of RTW among workers with acute LBP. This was confirmed in a later review of RTW among people with non-chronic non-specific LBP. ${ }^{17}$ Hartvigsen et $a l^{86}$ reviewed the evidence of psychosocial factors at work being important for RTW among people with LBP, and concluded that there is insufficient evidence of it.

To our knowledge, previous studies on factors affecting RTW among people with long-term (ie, not acute) pain in the neck/shoulder or back have not been systematically reviewed. It is possible that these factors differ from factors considered important for RTW among people with acute pain. Therefore, the aim of the present systematic review was to narratively summarise prognostic factors for RTW among people with long-term neck/shoulder or back pain.

\section{METHODS}

\section{Identification of studies}

We performed an extensive search through each of the databases Medline, CINAHL and PsycINFO from its inception until February 2016 for observational studies published in English of prognostic factors for RTW among people with pain in the neck/shoulder or back. A detailed description of the search terms and search strategy is presented in the online supplementary appendix 1 . The terms used in the search were defined based on the ideas behind the PICO model. ${ }^{37}$ We selected suitable keywords for $\mathrm{P}$ (population), I (intervention), $\mathrm{O}$ (outcome), while $\mathrm{C}$ (comparison) was excluded since comparison studies were not the focus for this study. Afterwards, the results from the three databases were combined. The reference list for each selected study was screened for additional relevant studies. Moreover, a search for studies that have cited each selected study was performed using the Scopus database, and reviews or meta-analyses were screened for relevant references or included studies.

\section{Selection of studies}

All authors independently screened the titles, abstracts and, if necessary, the full text of the articles for eligibility based on the inclusion criteria:

- subjects' age between 18 and 65 years

- work absence $\geq 2$ weeks

- neck/shoulder or back pain.

Work absence was defined as part-time or full-time absenteeism from work. The lower limit of 2 weeks was set to avoid inclusion of studies on prognostic factors related to acute injury or trauma, since they might differ substantially from prognostic factors related to long-term pain. Accordingly, long-term pain in the neck, shoulder or back was defined as pain that was not attributed to acute injury or trauma requiring at least 2 weeks part-time or full-time absenteeism from work. The exclusion criteria were:

- outcome not RTW

\begin{tabular}{ll}
\hline Table 1 & Quality assessment criteria \\
\hline 1 & Did the sample represent the population of interest? \\
2 & Was loss to follow-up $<35 \%$ and response rate $>65 \% ?$ \\
3 & $\begin{array}{l}\text { Were the prognostic factors measured with valid and } \\
\text { reliable instruments? }\end{array}$ \\
4 & Was the outcome of the study objectively measured? \\
5 & $\begin{array}{l}\text { Were important potential confounders (ie, age, gender, } \\
\text { prior WA and comorbidity) appropriately accounted for } \\
\text { in the analysis? }\end{array}$ \\
6 & Was the statistical analysis appropriate?
\end{tabular}

WA, work absence.

- non-observational study (ie, review/meta-analysis, intervention study or clinical trial)

- confounding rehabilitation programme

- instrumental validation

- population partly consisting of subjects fitting the inclusion criteria but for whom the results were not reported specifically.

Return to work can be described as an individual's cognitive and behavioural response to no longer being absent from work due to sickness. ${ }^{38}$ Consequently, it can be measured in different ways. In the present review, RTW was defined as being back at work (part-time or full-time) for at least 1 day. Following this definition, any indicator of work resumption was acceptable.

\section{Quality assessment}

Two reviewers (MR, MH) independently assessed the methodological quality of the included studies using a set of criteria that was adapted from 'guidelines for assessing the quality in prognostic studies on the basis of Framework of Potential Biases. ${ }^{39}$ It included six quality assessment criteria with focus on the key areas of potential bias in prognostic studies: study population, study attrition, measurement of prognostic factors, measurement of outcomes, measurement of and controlling for confounding variables, and analysis approaches (table 1). In the second criterion, the limits for response rate and loss to follow-up were set to $65 \%$ and $35 \%$, respectively. They were considered relevant in light of declining participation rates in epidemiologic studies, and have been used in previous health-related research. ${ }^{40}$ In the fifth criterion, we considered age, gender, prior work absence and comorbidity as potentially important confounders to be accounted for in the analysis, as their association with RTW in people with neck/shoulder or back pain has previously been demonstrated. ${ }^{23} 2642-44$

The reviewers graded each criterion as yes $=2$, partly $=1$ or no/unclear $=0$ on the basis of information provided in the articles. Criterion 1 (study population) was graded 'partly' if a subgroup of the population fulfilled our inclusion criteria. Criterion 2 (study attrition) was graded 'partly' if either the response rate exceeded $65 \%$ or the 
loss to follow-up was less than 35\%. Criterion 3 (prognostic factors) was graded 'partly' if the validity and/or reliability was reported for some, but not all, measurements of prognostic factors.

Criterion 4 (outcomes) was graded 'partly' if some, but not all, outcomes were measured objectively. Criterion 5 (confounders) was graded 'partly' if some, but not all, listed variables were controlled for in the analysis. Criterion 6 (analysis) was graded 'yes' if a multiple regression model corresponding to the outcome measurement was used and the predictors were selected without relying on empirical information about the measured exposure, ${ }^{45}$ 'partly' if a multiple regression model corresponding to the outcomes measurement was used and stepwise or bivariate statistical analysis of each potential predictor's association with the outcome was used to guide the selection of predictors in the model, and 'no/unclear' otherwise. In case of disagreement between the two reviewers, remaining reviewers also reviewed the article, and the judgement made by the majority of the reviewers determined the quality rating. A quality score for each study was calculated as the sum of all scores, thus ranging between 0 and 12 points where higher scores indicate better quality. No weighting was used, as we did not consider any area of potential bias to be more important than another.

\section{Data synthesis}

We identified main factors that were shown to be significantly $(\mathrm{p}<0.05)$ or non-significantly associated with RTW in the included studies. Factors that expressed related meanings, that is, based on similarities, were grouped together into categories. Afterwards, each category was labelled according to the factors investigated in the studies.

\section{RESULTS}

A flow diagram of the selection process is shown in figure 1. In total, 769 studies were identified from the databases search. After removing the duplicates, 333 studies remained. Out of these, 51 were considered eligible based on the title and abstract. When screening the 51 full texts, 7 studies met all of the inclusion criteria (see online supplementary appendix 2). In the additional search through reference lists and citations of the included studies, three studies fulfilled the inclusion criteria. As a result, a total of 10 studies $^{46-55}$ remained for methodological quality assessment and synthesis of results.

These 10 studies originated from Sweden, Belgium, the USA, Canada and the Netherlands (table 2). All of them were longitudinal prospective cohort studies with a follow-up of 3-24 months, except one that was retrospective. They were conducted on populations with pain in the neck or low back that had been absent from work for at least 4 weeks. Table 2 describes each of the selected studies in terms of study design, study population, sample size, attrition and factors identified as significant and non-significant, respectively, in relation to the outcome RTW.

\section{Quality scores}

For the 10 selected studies, the quality scores ranged from 4 to 9 points. The studies were classified as low quality ( $0-4$ points), medium quality (5-8 points) and high quality (9-12 points) (table 3 ).

In the synthesis of results, five categories of the factors were extracted: recovery beliefs, health-related factors, workplace factors, work capacity and behaviour (table 4). Each of them is described in detail below.

\section{Recovery beliefs}

Recovery beliefs comprise many aspects, such as believing that you will be able to function or work in the presence or absence of pain, or that you will be in control of your situation. ${ }^{56}$ They were evaluated as predictors of RTW in two low and two medium-quality studies. ${ }^{46} 485154$ All studies reported a significant positive association between recovery beliefs and RTW, when the predictor was measured by the Expectations of Recovery Scale, the Work-related Recovery Expectations Questionnaire, and single questions, respectively (table 4).

\section{Health-related factors}

Health is a multifaceted concept, and more than merely the absence of disease ${ }^{57}$ It involves several dimensions, for example, quality of life and vitality. It can also be separated into physical and mental components. Among the reviewed studies, the association between health-related factors and RTW was investigated in four studies of low and medium qualities. The medium-quality studies consistently reported that health, in terms of health-related quality of life, health transition and general health, was a significant predictor of RTW. ${ }^{52} 5455$ In the low-quality study, no significant association between vitality, mental health and RTW was found. ${ }^{48}$ Table 4 shows the instruments used to measure the health-related factors.

\section{Workplace factors}

Three medium-quality studies investigated the association between workplace factors and RTW. Two of them showed that type of work (blue colour work) and job satisfaction, respectively, significantly related to $\mathrm{RTW}^{49}{ }^{55}$ whereas one study found no significant association between coworker support and RTW. ${ }^{54}$ The different predictors were measured using single questions and the Job Satisfaction Scale (table 4).

\section{Work capacity}

Several studies investigated the predictive ability of factors related to work capacity for RTW. Among them, two medium-quality studies found that pain intensity and prior pain duration were significantly associated with RTW. ${ }^{45}$ In one low-quality and two medium-quality studies, selfrated disability was also identified as a significant 


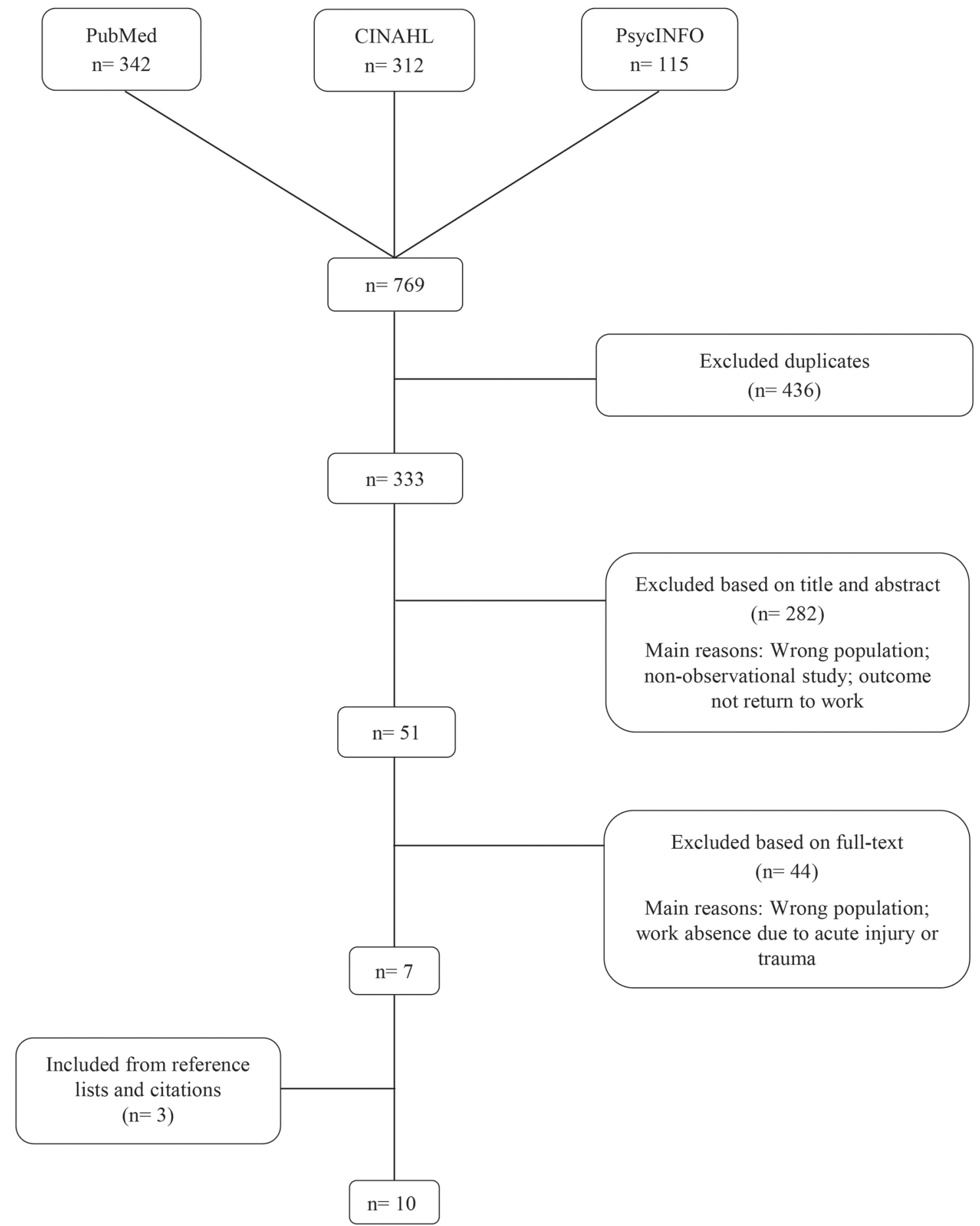

Figure 1 Flow diagram of the selection process. All authors independently screened the titles, abstracts and, if necessary, the full text of the articles.

predictor of RTW. ${ }^{48}{ }^{52}$ Work ability, measured by the Work Ability Index, contributed significantly to RTW in one medium-quality study. ${ }^{53}$ One high-quality and one medium-quality study investigated the prognostic value of functional capacity tests for RTW, and found different results. ${ }^{50}$ In the high-quality study, findings from functional capacity lifting tests significantly predicted RTW, whereas in the medium-quality study, findings from functional capacity evaluation involving several tasks showed no significant association with RTW. More information about the measurement of the factors is presented in table 4.

\section{Behaviour}

Pain behaviour and fear avoidance beliefs were investigated in one medium-quality study, where both were identified as significant predictors for RTW. ${ }^{49}$ In the study, pain behaviour was measured by the Pain Behaviour Scale, and fear avoidance beliefs with the Fear Avoidance Beliefs Questionnaire (table 4). 


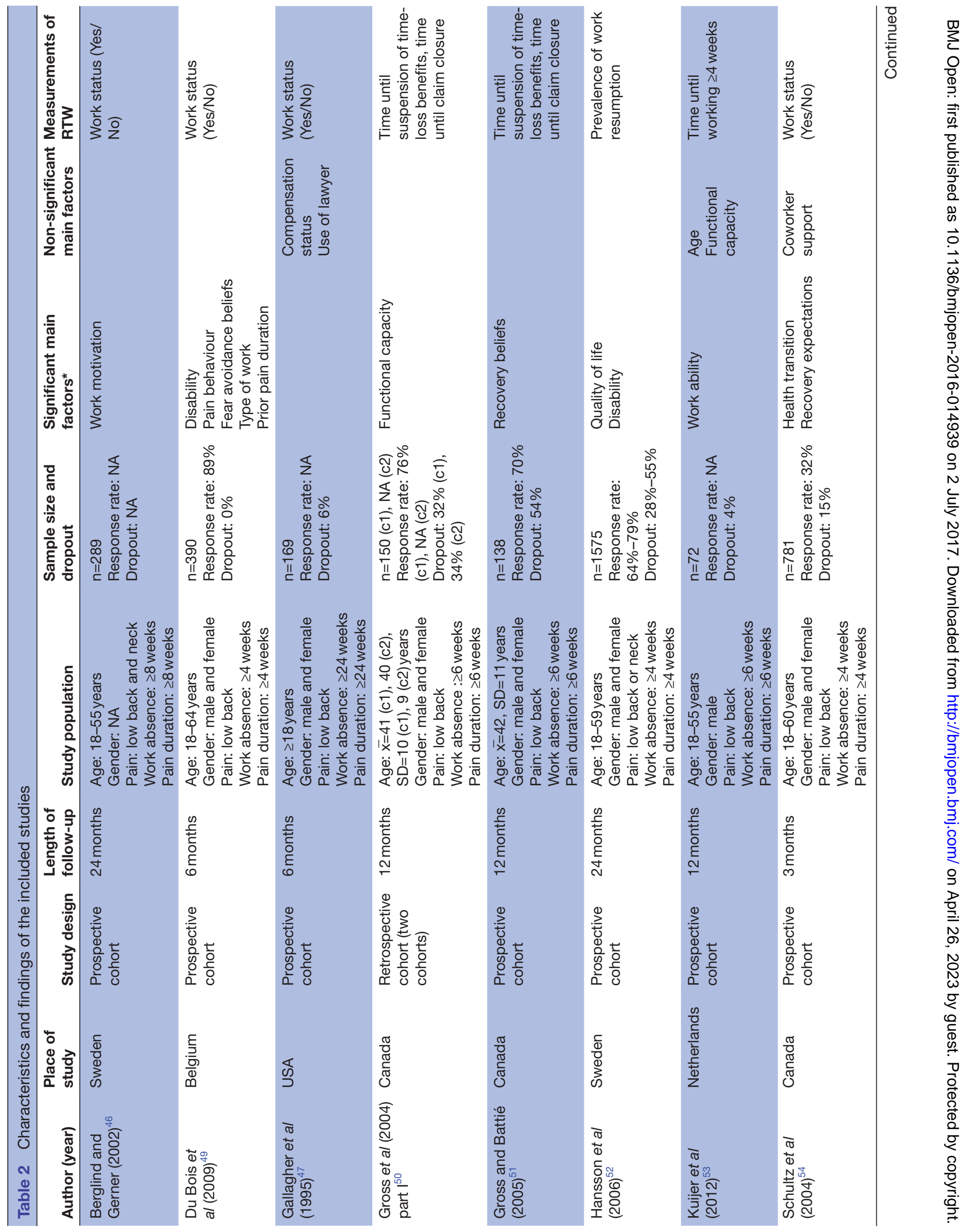



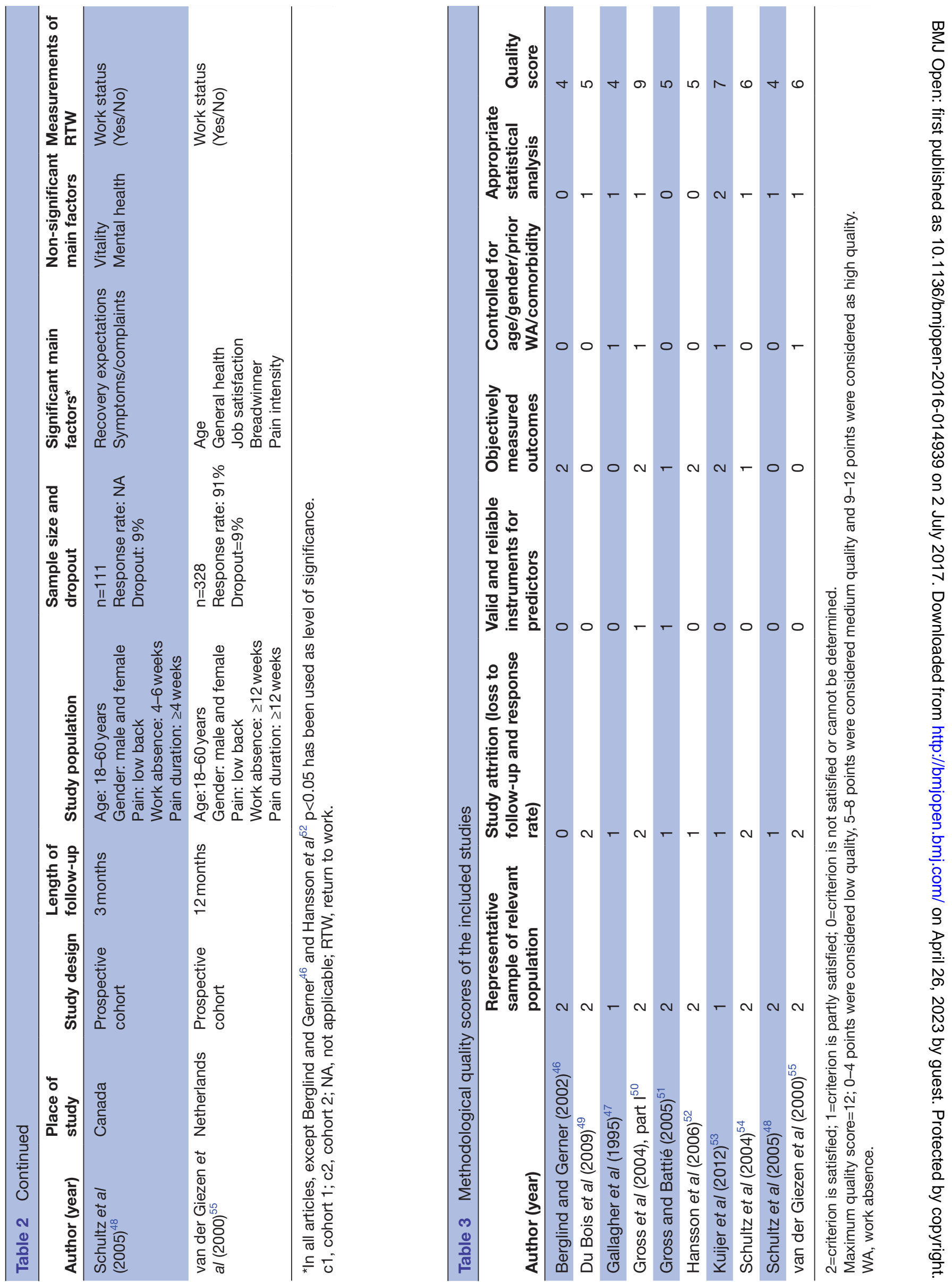


\begin{tabular}{|c|c|c|}
\hline Factors & Measurement & Categories \\
\hline Recovery beliefs ${ }^{4651}$ & Expectations of Recovery Scale, single questions & Recovery beliefs \\
\hline Quality of life ${ }^{52}$ & EuroQol & Health-related factors \\
\hline Health transition ${ }^{54}$ & Short Form (36) Health Survey & \\
\hline Mental health ${ }^{48}$ & Short Form (36) Health Survey & \\
\hline General health ${ }^{55}$ & Short Form (36) Health Survey & \\
\hline Type of work ${ }^{49}$ & Single question & Workplace factors \\
\hline Coworker support ${ }^{54}$ & Single question & \\
\hline Job satisfaction ${ }^{55}$ & Job Satisfaction Scale & \\
\hline Work ability ${ }^{53}$ & Work Ability Index & \\
\hline Pain intensity ${ }^{55}$ & Pain Complaint Questionnaire & \\
\hline Symptoms/complaints ${ }^{48}$ & Single questions & \\
\hline Pain behaviour ${ }^{49}$ & Pain Behaviour Scale & Behaviour \\
\hline Fear avoidance beliefs ${ }^{49}$ & Fear Avoidance Beliefs Questionnaire & \\
\hline
\end{tabular}

EuroQol, European Quality of Life Scale.

Gallagher et al. (1995) was not included since the factors Compensation status and Use of lawyer could not readily be categorized with other factors.

\section{DISCUSSION}

In the present systematic review, we synthesised the results from observational studies of prognostic factors for RTW among people with long-term neck or back pain. A total of 10 studies were included from the literature search. Among them, one was classified as high quality, six as medium quality and three as low-quality studies according to our quality assessment criteria. From the studies, five categories of factors were extracted: recovery beliefs, health-related factors, workplace factors, work capacity and behaviour.

\section{Recovery beliefs}

Our findings, suggesting that recovery beliefs are important for RTW, are consistent with previous reviews of people with back pain showing that recovery beliefs are associated with better health outcomes and RTW. ${ }^{1722}$ One possible explanation for recovery beliefs being related to RTW is that when people believe that they will not recover from the illness, they may experience lower competence and motivation for returning to work. ${ }^{17} 2158$ The fact that simple measurements of recovery beliefs can be used to predict RTW may be useful in practice, when determining which treatment/rehabilitation to apply.

\section{Health-related factors}

Previous reviews of people with acute, subacute and non-specific $\mathrm{LBP}^{17} 59$ have shown that health is an important predictor of RTW. Among the studies included in this review, the results were not entirely consistent. Considering the quality of the studies, and the different aspects of health investigated, it appears that health-related factors should be paid attention to in relation to RTW. It seems reasonable that perceived health is positively associated with RTW, since healthy people are more likely to feel capable of working. ${ }^{202360}$ For rehabilitation purposes, however, more information about which aspects of health are important for RTW is needed to provide targeted interventions for reducing work absence among people with long-term neck or back pain. To achieve this, more in-depth analyses of components of health that are important for RTW are needed.

\section{Workplace factors}

The diversity in results as well as workplace factors investigated in the studies prevents any solid conclusions from being drawn. While it is conceivable that type of work performed is important for RTW, it would likely depend on which types of work are considered, and it is not clear how this information could be useful in practice without more knowledge about the work demands involved. Previous reviews have found that job satisfaction cannot predict failure to RTW in patients with non-chronic non-specific $\mathrm{LBP},{ }^{17}$ and that coworker support is important for work disability following low back injury. ${ }^{161}$ Taken together, our results concerning the importance of workplace factors for RTW among people with long-term neck or 
back pain are inconclusive. More research is needed to confirm or refute this.

\section{Work capacity}

Among the studies of factors related to work capacity, the findings were mostly consistent. While self-rated measures of work capacity in terms of pain, disability or work ability can significantly predict RTW among people with longterm pain in the neck or back, the ability of functional capacity evaluation tests to do so may be affected by how the tests are performed. Our results are in agreement with previous reviews. For example, Crook $e t a b^{62}$ found that functional disability was an important prognostic factor of work outcome for people with low-back injury, and Verkerk et $a t^{63}$ found evidence of a positive influence of lower pain intensity on RTW among people with non-specific LBP. It appears, therefore, that work capacity is important to be considered in the treatment/rehabilitation of people with MSDs in the neck or back.

\section{Behaviour}

Since factors relating to behaviour were investigated in only one study, no solid conclusions about its relation to RTW in this population can be drawn. Previous reviews are also inconsistent. Iles et $a l^{17}$ concluded that fear avoidance beliefs are predictive of RTW among people with non-chronic non-specific LBP. However, Pincus et $a b^{64}$ found little evidence to link fear of pain with poor outcome in acute LBP. This discrepancy may be due to differences in the populations studied and/or in the methods used for assessing the factors.

In two of the included studies, the impact of age on RTW was reported. ${ }^{53} 55$ In most studies, however, age is treated as a confounding factor, and the significance of it is not specifically reported. For that reason, and the fact that it cannot be targeted in treatment/rehabilitation, we have refrained from drawing conclusions from the reported findings. Interestingly, the study by van der

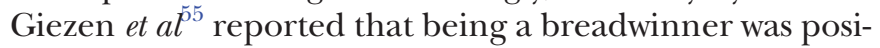
tively associated with RTW among people with LBP. As it is the only study that has addressed the importance of responsibilities towards others in returning to work, no solid conclusions can be drawn about its importance. However, it may be an important factor to adjust for in future studies of RTW among people with MSDs.

\section{Methodological considerations regarding the articles in the review}

In most of the studies we retrieved during our literature search, no distinction was made between sick leave, absenteeism, work absence and time out of work. These are not standardised terms, but rather vary from country to country. For consistency, we used work absence throughout the paper.

Our definition of RTW allowed it to be measured differently in the studies. Some studies based it on records ${ }^{465152}$ and others on self-report, ${ }^{49} 511^{53-55}$ and the studies differed in the duration and proportion of work needed to be defined as RTW. It is possible that more consistent use of definitions across studies would have affected the results obtained in the present study.

As effect sizes were not reported consistently in the studies, our conclusions were based on the information provided concerning the significant and non-significant factors for RTW. Only one study received a high-quality score based on the six methodological criteria for assessing the quality of prognostic studies. Surprisingly, no study reported the statistical power of the analysis performed, and in most of the studies the validity and reliability of the instruments used was not reported.

\section{Strengths and limitations of the systematic review}

Previous reviews on prognostic factors of RTW have focused largely on people with acute pain. The present review highlights predictors for RTW among people with long-term pain in the neck or back by considering observational studies only. Thereby, it contributes to previous findings by addressing factors of importance for patients with long lasting pain in their natural course of RTW.

The strength of the present systematic review is that we searched for studies in three databases covering a wide range of research papers, and that we used a long time interval for each of the databases so as to access as much as possible of relevant literature. We used clear inclusion and exclusion criteria with respect to the population, exposures and study outcome. We also assessed the quality of the studies and confirmed our findings among the authors. Only observational studies were included to minimise potential sources of bias and study the natural course of RTW. The quality of the studies was assessed by considering six important potential sources of bias in studies of prognostic factors. ${ }^{39}$ Since we considered them to be equally important for study quality, they were summarised into a single quality score. The quality score was then divided into tertiles to indicate the level of quality (low/medium/high) of the studies, thereby allowing quality as well as quantity to be considered in the synthesis of results.

One potential limitation is that only journals publishing studies in English were included, which may have led to the exclusion of important studies from our search. Furthermore, the small number of published studies on prognostic factors for RTW among people with long-term neck or back pain prevents us from drawing solid conclusions concerning predictive factors of RTW in this population. Moreover, as most of the included studies concerned LBP only, our results may be less applicable to people with neck pain. Although pain patients were investigated in all included studies, the aetiology of the pain was likely different. Some of the studies reported the origin of the pain, ${ }^{49}{ }^{50}$ but others did not do so specifically. ${ }^{52}$ This may have affected our results. 


\section{CONCLUSION}

In the present review, we identified five categories of factors from studies of RTW among people absent from work ( $\geq 2$ weeks) due to long-term neck or back pain. Our results indicate that recovery beliefs, health-related factors and work capacity are important for RTW in this population. However, few studies have been conducted on this population, and the quality of the studies was generally not high. Thus, we call for more high-quality prospective studies that can improve our understanding of what is needed to facilitate RTW for people with long-term neck or back pain.

Acknowledgements We would like to express our gratitude and appreciation to the librarian Malin Almstedt Jansson for assisting us in developing the search strategy.

Contributors MR codeveloped the search strategy, performed the search, screened and quality assessed the articles, and drafted the manuscript. MLK codeveloped the search strategy, screened articles, contributed to the quality assessment of the articles and reviewed the initial drafts of the manuscript. AN codeveloped the search strategy, screened articles, contributed to the quality assessment of the articles and reviewed the initial drafts of the manuscript. $\mathrm{MH}$ conceived the study, codeveloped the search strategy, screened and quality assessed the articles, and reviewed the initial drafts of the manuscript. All authors have read and approved the final manuscript.

Funding This project was supported by University of Gävle (Dnr. 2015/424). Competing interests None declared.

Provenance and peer review Not commissioned; externally peer reviewed.

Data sharing statement The data sets generated during and/or analysed during the current study are available from the corresponding author upon request.

Open Access This is an Open Access article distributed in accordance with the Creative Commons Attribution Non Commercial (CC BY-NC 4.0) license, which permits others to distribute, remix, adapt, build upon this work non-commercially, and license their derivative works on different terms, provided the original work is properly cited and the use is non-commercial. See: http://creativecommons.org/ licenses/by-nc/4.0/

(c) Article author(s) (or their employer(s) unless otherwise stated in the text of the article) 2017. All rights reserved. No commercial use is permitted unless otherwise expressly granted.

\section{REFERENCES}

1. Henderson M, Glozier N, Holland Elliott K. Long term sickness absence: Is caused by common conditions and needs managing. BMJ 2005;330:802-3.

2. Frank JW, Brooker AS, DeMaio SE, et al. Disability resulting from occupational low back pain. Part II: what do we know about secondary prevention? A review of the scientific evidence on prevention after disability begins. Spine 1996;21:2918-29.

3. Abenhaim L, Rossignol M, Gobeille D, et al. The prognostic consequences in the making of the initial medical diagnosis of workrelated back injuries. Spine 1995;20:791-5

4. Alexanderson K. Sickness absence: a review of performed studies with focused on levels of exposures and theories utilized. Scand J Soc Med 1998;26:241-9.

5. Lancourt J, Kettelhut M. Predicting return to work for lower back pain patients receiving worker's compensation. Spine 1992;17:629-40.

6. Ihlebaek $\mathrm{C}$, Hansson $\mathrm{TH}$, Laerum $\mathrm{E}$, et al. Prevalence of low back pain and sickness absence: a "borderline" study in Norway and Sweden. Scand J Public Health 2006;34:555-8.

7. Besen E, Young AE, Shaw WS. Returning to work following low back pain: towards a model of individual psychosocial factors. J Occup Rehabil 2015;25:25-37.

8. Gallagher RM, Rauh V, Haugh LD, et al. Determinants of return-towork among low back pain patients. Pain 1989;39:55-67.

9. Kool JP, Oesch PR, de Bie RA. Predictive tests for non-return to work in patients with chronic low back pain. Eur Spine J 2002;11:258-66.
10. Nyman T, Grooten WJ, Wiktorin C, et al. Sickness absence and concurrent low back and neck-shoulder pain: results from the MUSIC-Norrtälje study. Eur Spine J 2007;16:631-8.

11. Leboeuf-Yde C, Fejer R, Nielsen J, et al. Consequences of spinal pain: do age and gender matter? A Danish cross-sectional population-based study of 34,902 individuals 20-71 years of age. BMC Musculoskelet Disord 2011;12:39.

12. Borg K, Hensing G, Alexanderson K. Prediction of future low levels of sickness absence among young persons sick listed with back, neck, or shoulder diagnoses. Work 2004;23:159-67.

13. Waddell G. Preventing incapacity in people with musculoskeletal disorders. Br Med Bull 2006;78:55-69.

14. Ahrberg $Y$, Landstad BJ, Bergroth $A$, et al. Desire, longing and vanity: emotions behind successful return to work for women on long-term sick leave. Work 2010;37:167-77.

15. Hansson T, Jensen I. Swedish Council on Technology Assessment in Health Care (SBU). Chapter 6. sickness absence due to back and neck disorders. Scand J Public Health Supp/ 2004;63:109-51.

16. Dekkers-Sánchez PM, Hoving JL, Sluiter JK, et al. Factors associated with long-term sick leave in sick-listed employees: a systematic review. Occup Environ Med 2008;65:153-7.

17. Iles RA, Davidson M, Taylor NF. Psychosocial predictors of failure to return to work in non-chronic non-specific low back pain: a systematic review. Occup Environ Med 2008;65:507-17.

18. Josephson M, Heijbel B, Voss M, et al. Influence of self-reported work conditions and health on full, partial and no return to work after long-term sickness absence. Scand J Work Environ Health 2008;34:430-7.

19. Gustafsson K, Lundh G, Svedberg P, et al. Psychological factors are related to return to work among long-term sickness absentees who have undergone a multidisciplinary medical assessment. J Rehabil Med 2013;45:186-91.

20. Steenstra IA, Verbeek JH, Heymans MW, et al. Prognostic factors for duration of sick leave in patients sick listed with acute low back pain: a systematic review of the literature. Occup Environ Med 2005;62:851-60.

21. Heymans MW, de Vet HC, Knol DL, et al. Workers' beliefs and expectations affect return to work over 12 months. J Occup Rehabil 2006;16:685-95.

22. Mondloch MV, Cole DC, Frank JW. Does how you do depend on how you think you'll do? A systematic review of the evidence for a relation between patients' recovery expectations and health outcomes. CMAJ 2001;165:174-9.

23. Burdorf A, Naaktgeboren B, Post W. Prognostic factors for musculoskeletal sickness absence and return to work among welders and metal workers. Occup Environ Med 1998;55:490-5.

24. Linton SJ. Occupational psychological factors increase the risk for back pain: a systematic review. J Occup Rehabil 2001;11:53-66.

25. Dunstan DA, MacEachen E. Bearing the brunt: co-workers' experiences of work reintegration processes. J Occup Rehabil 2013;23:44-54.

26. Alexopoulos EC, Konstantinou EC, Bakoyannis G, et al. Risk factors for sickness absence due to low back pain and prognostic factors for return to work in a cohort of shipyard workers. Eur Spine $J$ 2008;17:1185-92.

27. Gouttebarge V, Kuijer PP, Wind $\mathrm{H}$, et al. Criterion-related validity of functional capacity evaluation lifting tests on future work disability risk and return to work in the construction industry. Occup Environ Med 2009;66:657-63.

28. Hagen EM, Svensen E, Eriksen HR. Predictors and modifiers of treatment effect influencing sick leave in subacute low back pain patients. Spine 2005;30:2717-23.

29. Steenstra IA, Ibrahim SA, Franche RL, et al. Validation of a risk factorbased intervention strategy model using data from the readiness for return to work cohort study. J Occup Rehabil 2010;20:394-405.

30. Alexanderson KA, Borg KE, Hensing GK. Sickness absence with lowback, shoulder, or neck diagnoses: an 11-year follow-up regarding gender differences in sickness absence and disability pension. Work 2005;25:115-24.

31. Storheim K, Brox JI, Holm I, et al. Predictors of return to work in patients sick listed for sub-acute low back pain: a 12-month followup study. J Rehabil Med 2005;37:365-71.

32. Eshøj P, Jepsen JR, Nielsen CV. Long-term sickness absence - risk indicators among occupationally active residents of a danish county. Occup Med 2001;51:347-53.

33. Allebeck P, Mastekaasa A. Swedish Council on Technology Assessment in Health Care (SBU). Chapter 5. risk factors for sick leave - general studies. Scand J Public Health Supp/ 2004;63:49-108. 
34. Borg K, Hensing G, Alexanderson K. Risk factors for disability pension over 11 years in a cohort of young persons initially sicklisted with low back, neck, or shoulder diagnoses. Scand J Public Health 2004;32:272-8.

35. Fritz JM, Wainner RS, Hicks GE. The use of nonorganic signs and symptoms as a screening tool for return-to-work in patients with acute low back pain. Spine 2000;25:1925-31.

36. Hartvigsen J, Lings S, Leboeuf-Yde C, et al. Psychosocial factors at work in relation to low back pain and consequences of low back pain; a systematic, critical review of prospective cohort studies. Occup Environ Med 2004;61:e2.

37. Cooke A, Smith D, Booth A. Beyond PICO: the SPIDER tool for qualitative evidence synthesis. Qual Health Res 2012;22:1435-43.

38. Wasiak R, Young AE, Roessler RT, et al. Measuring return to work. $J$ Occup Rehabil 2007;17:766-81.

39. Hayden JA, Côté P, Bombardier C. Evaluation of the quality of prognosis studies in systematic reviews. Ann Intern Med 2006;144:427-37.

40. Gold JE, Hallman DM, Hellström F, et al. Systematic review of biochemical biomarkers for neck and upper-extremity musculoskeletal disorders. Scand J Work Environ Health 2016;42:103-24.

41. Galea S, Tracy M. Participation rates in epidemiologic studies. Ann Epidemiol 2007;17:643-53.

42. Busch H, Göransson S, Melin B. Self-efficacy beliefs predict sustained long-term sick absenteeism in individuals with chronic musculoskeletal pain. Pain Pract 2007;7:234-40.

43. Okurowski L, Pransky G, Webster B, et al. Prediction of prolonged work disability in occupational low-back pain based on nurse case management data. J Occup Environ Med 2003;45:763-70.

44. Opsahl J, Eriksen HR, Tveito TH. Do expectancies of return to work and Job satisfaction predict actual return to work in workers with long lasting LBP? BMC Musculoskelet Disord 2016;17:481.

45. Heiden M, Mathiassen SE, Garza J, et al. A comparison of two strategies for building an exposure prediction Model. Ann Occup Hyg 2016;60:74-89.

46. Berglind $\mathrm{H}$, Gerner $\mathrm{U}$. Motivation and return to work among the long-term sicklisted: an action theory perspective. Disabil Rehabil 2002;24:719-26.

47. Gallagher RM, Williams RA, Skelly J, et al. Workers' Compensation and return-to-work in low back pain. Pain 1995;61:299-307.

48. Schultz IZ, Crook J, Berkowitz J, et al. Predicting return to work after low back injury using the Psychosocial risk for Occupational Disability Instrument: a validation study. J Occup Rehabil 2005;15:365-76.

49. Du Bois M, Szpalski M, Donceel P. Patients at risk for long-term sick leave because of low back pain. Spine J 2009;9:350-9.
50. Gross DP, Battié MC, Cassidy JD. The prognostic value of functional capacity evaluation in patients with chronic low back pain: part 1: timely return to work. Spine 2004;29:914-9.

51. Gross DP, Battié MC. Work-related recovery expectations and the prognosis of chronic low back pain within a workers' compensation setting. J Occup Environ Med 2005;47:428-33.

52. Hansson E, Hansson T, Jonsson R. Predictors for work ability and disability in men and women with low-back or neck problems. Eur Spine J 2006;15:780-93.

53. Kuijer PP, Gouttebarge V, Wind $\mathrm{H}$, et al. Prognostic value of self-reported work ability and performance-based lifting tests for sustainable return to work among construction workers. Scand $J$ Work Environ Health 2012;38:600-3.

54. Schultz IZ, Crook J, Meloche GR, et al. Psychosocial factors predictive of occupational low back disability: towards development of a return-to-work model. Pain 2004;107:77-85.

55. van der Giezen AM, Bouter LM, Nijhuis FJ. Prediction of returnto-work of low back pain patients sicklisted for 3-4 months. Pain 2000;87:285-94.

56. Hestbaek L, Leboeuf-Yde C, Manniche C. Low back pain: what is the long-term course? A review of studies of general patient populations. Eur Spine $J$ 2003;12:149-65.

57. Johansson H, Weinehall L, Emmelin M. "It depends on what you mean": a qualitative study of Swedish health professionals' views on health and health promotion. BMC Health Serv Res 2009;9:191.

58. Heijbel B, Josephson M, Jensen I, et al. Return to work expectation predicts work in chronic musculoskeletal and behavioral health disorders: prospective study with clinical implications. J Occup Rehabil 2006;16:169-80.

59. Schaafsma FG, Whelan K, van der Beek AJ, et al. Physical conditioning as part of a return to work strategy to reduce sickness absence for workers with back pain. Cochrane Database Syst Rev 2013;8:CD001822.

60. Linton SJ, Halldén K. Can we screen for problematic back pain? A screening questionnaire for predicting outcome in acute and subacute back pain. Clin J Pain 1998;14:209-15.

61. Shaw WS, Pransky G, Fitzgerald TE. Early prognosis for low back disability: intervention strategies for health care providers. Disabil Rehabil 2001;23:815-28.

62. Crook J, Milner R, Schultz IZ, et al. Determinants of occupational disability following a low back injury: a critical review of the literature. J Occup Rehabil 2002;12:277-95.

63. Verkerk K, Luijsterburg PA, Miedema HS, et al. Prognostic factors for recovery in chronic nonspecific low back pain: a systematic review. Phys Ther 2012;92:1093-108.

64. Pincus T, Vogel S, Burton AK, et al. Fear avoidance and prognosis in back pain: a systematic review and synthesis of current evidence. Arthritis Rheum 2006;54:3999-4010. 